\title{
Control of Linear Time-Varying Systems by The Gradient Algorithm
}

\author{
Min-Shin Chen \\ Department of Mechanical Engineering \\ National Taiwan University \\ Taipei, Taipei, R.O.C. \\ mschen@ccms.ntu.edu.tw
}

\begin{abstract}
In this paper, a new control design is proposed for a linear time-varying system which exhibits sustained but bounded state oscillations. The design is based on the gradient algorithm used in the adaptive parameter identification. The resultant observer-based state feedback control guarantees exponential decay of the state oscillation given that the system is both uniformly controllable and uniformly observable. A unique feature of the proposed control design is that it requires neither information of time derivatives of the time-varying parameters, nor prediction of future information of the time-varying parameters.
\end{abstract}

\section{Introduction}

For linear time-varying systems, particularly for those with periodically time-varying system parameters, there have been a variety of control designs proposed. Examples can be found in [1,3,6,11]. For systems with non-periodically time-varying parameters, the pole-placement like control in $[5,12,13]$ provides a sound theoretical solution to the control problem. However, their control designs require time derivatives of the parameters up to the order of the system dimension. Since it is impossible to have "clean" measurement of the time-varying parameters, the measurement noise will inevitably be amplified in the time differentiation process, thus causing noticeable state oscillations in the closed-loop response. Other control designs include the LQ optimal control [7] and the controllability-grammian-based control [4,9]. However, they suffer from the disadvantage that the control engineers must be able to predict how the time-varying parameters vary in the future in order to calculate the desired control input at any time instant.
In this paper, a ne'w design is presented for the control of a non-periodically time-varying system whose state exhibits sustained but bounded oscillations. In this new approach, one uses the open-loop state transition matrix to transform the system into one with a zero system matrix, and then utilizes the "gradient algorithm" in adaptive parameter identification [8] to synthesize a stabilizing control. The same approach also leads to an observer: design that asymptotically recovers the system state from the system output when full state measurement is not available. The resultant observer-based state feedback control can be applied to systems with periodically or non-periodically timevarying parameters. Most importantly, it offers two advantages over other controls: (1) There is no need to predict future information of the time-varying parmeters. The control design requires only past and present information of the parameters. (2) There is no need to take time derivatives of the time-varying parameters. This avoids the noise amplification problem in the pole-placement like control.

\section{Problem Formulation}

Consider a multivariable linear time-varying system

$$
\begin{aligned}
& \dot{x}(t)=A(t) x(t)+B(t) u(t), \quad x(0)=x_{0} \\
& y(t)=C(t) x(t),
\end{aligned}
$$

where $x(t) \in R^{n}$ is the system state vector, $u(t) \in R^{m}$ is the control input, and $y(t) \in R^{p}$ is the system output. The system matrix $A(t) \in R^{n \times n}$, the input matrix $B(t) \in R^{n \times m}$, and the output matrix $C(t) \in R^{p \times n}$ are time-varying matrices whose elements are bounded, piecewise continuous functions of time. Notice that $A(t), B(t)$ and $C(t)$ may be non-periodically timevarying. It is assumed that the open-loop state trajectory $x(t)$ exhibits sustained but bounded oscillations. 
In other words, the state transition matrix $\Phi\left(t, t_{0}\right) \in$ $R^{n \times n}$ of the open-loop system remains bounded and nonzero for all $t$ and $t_{0}$. Therefore, there exist two positive constants $m_{1}$ and $m_{2}$ such that for all $t$ and $t_{0}$,

$$
m_{1} \leq \sigma_{i}\left[\Phi\left(t, t_{0}\right)\right] \leq m_{2}, \quad \forall i=1,2, \ldots, n
$$

where $\sigma_{i}$ denotes the singular value of a matrix, and the state transition matrix [2] $\Phi\left(t, t_{0}\right)$ is defined by

$$
\begin{aligned}
\frac{\partial \Phi\left(t, t_{0}\right)}{\partial t} & =A(t) \Phi\left(t, t_{0}\right), \quad \Phi(t, t)=I, \\
\Phi\left(t_{0}, t\right) & =\Phi^{-1}\left(t, t_{0}\right) .
\end{aligned}
$$

The objective of this paper is to find a control to suppress the oscillatory behavior of the open-loop system under the condition that the only accessible signal in the system is the system output $y(t)$. In particular, an observer-based state feedback control

$$
u(t)=-K(t) \hat{x}(t)
$$

is considered, where $\hat{x}(t)$ is an estimate of the state $x(t)$. For the existence of a stabilizing control as in Eq.(4), it is assumed that the system (1) is uniformly controllable as well as uniformly observable as defined below [9].

Definition 1 : The pair $(A(t), B(t))$ is uniformly controllable if there exist $\Delta, \beta_{1}$ and $\beta_{2} \in R^{+}$such that for all $t>0$,

$$
\beta_{1} I \leq P_{c}(t) \leq \beta_{2} I
$$

where $P_{c}(t) \in R^{n \times n}$ is the controllability grammian defined by

$$
P_{c}(t) \triangleq \int_{t-\Delta}^{t} \Phi(t-\Delta, \tau) B(\tau) B^{T}(\tau) \Phi^{T}(t-\Delta, \tau) d \tau,
$$

in which $\Phi(t, \tau)$ is the state transition matrix defined in Eq.(3).

Definition 2 : The pair $(A(t), C(t))$ is uniformly observable if there exist $\Delta, \gamma_{1}$ and $\gamma_{2} \in R^{+}$such that for all $t>0$,

$$
\gamma_{1} I \leq P_{o}(t) \leq \gamma_{2} I
$$

where $P_{o}(t) \in R^{n \times n}$ is the observability grammian defined by

$$
P_{o}(t) \triangleq \int_{t-\Delta}^{t} \Phi^{T}(\tau, t-\Delta) C^{T}(\tau) C(\tau) \Phi(\tau, t-\Delta) d \tau .
$$

\section{Preliminary}

The control and observer designs in this paper will utilize the well-known "gradient algorithm" originally developed in the adaptive parameter identification problem. Consider the parameter estimation error dynamics of a gradient algorithm:

$$
\dot{z}(t)=-\gamma w(t) w^{T}(t) z(t), \quad z(t) \in R^{n},
$$

where $\gamma$ is any positive constant, $z(t)$ represents the parameter estimation error, and $w(t) \in R^{n \times m}$ is usually called the "regressor". The following theorem gives a sufficient condition on the exponential stability of the system (7).

Theorem 1 : If the regressor $w(t)$ is "persistent exciting" in the sense that there exist positive constants $\Delta, \alpha_{1}$, and $\alpha_{2}$ such that

$$
\alpha_{1} I \leq \int_{t-\Delta}^{t} w^{T}(\tau) w(\tau) d \tau \leq \alpha_{2} I, \quad \forall t>0,
$$

then the system (8) is exponentially stable, and

$$
\|z(k \Delta)\|^{2} \leq \rho^{k}\|z(0)\|^{2}, \quad k=1,2, \ldots,
$$

where $0<\rho<1$, and

$$
\rho=1-2 \sigma \gamma, \quad \sigma=\frac{\alpha_{1}}{\left(1+\gamma \alpha_{2} \sqrt{n}\right)^{2}} .
$$

Proof : see proof of Theorem 2.5.1 in [10].

\section{State Feedback Control Design}

In this Section it is temporarily assumed that the system state $x(t)$ is accessible, and the goal is to find a state feedback control

$$
u(t)=\cdots K(t) x(t)
$$

such that the closed-loop system (1) and (11) is exponentially stable. The proposed control design starts with a coordinate transformation

$$
x(t)=\Phi\left(t, t_{0}\right) z(t),
$$

where $z(t)$ is the new state coordinate, and the transformation matrix is exactly the open-loop state transition matrix $\Phi\left(t, t_{0}\right)$ in Eq.(3). Note that $\Phi\left(t, t_{0}\right)$ is always invertible by Theorem $4-2$ in [2]. Since $\Phi\left(t, t_{0}\right)$ is uniformly bounded as assumed in Eq.(2), the transformation (12) converts the stabilization problem of the 
system state $x(t)$ into that of the new state $z(t)$. According to Eqs.(1), (3) and (12), the governing equation of the new state $z(t)$ is given by

$$
\dot{z}(t)=\Phi\left(t, t_{0}\right)^{-1} B(t) u(t) .
$$

Note carefully that in this new coordinate, the system matrix is identically zero. For a system with a zero system matrix as in Eq.(13), Theorem 1 in the previous Section immediately suggests that the control $u(t)$ can be chosen as

$$
\begin{aligned}
u(t) & =-\gamma B^{T}(t) \Phi^{-T}\left(t, t_{0}\right) z(t) \\
& =-\gamma B^{T}(t) \Phi^{-T}\left(t, t_{0}\right) \Phi^{-1}\left(t, t_{0}\right) x(t)
\end{aligned}
$$

where $\gamma$ can be any positive constant. With this choice, the transformed closed-loop dynamics becomes

$$
\dot{z}(t)=-\gamma \Phi^{-1}\left(t, t_{0}\right) B(t) B^{T}(t) \Phi^{-T}\left(t, t_{0}\right) z(t),
$$

which has exactly the same structure as that in Eq.(7) with $\Phi^{-1}\left(t, t_{0}\right) B(t)$ acting as the regressor.

The following lemma shows that the persistent excitation condition on the regressor $\Phi^{-1}\left(t, t_{0}\right) B(t)$, which is required for the exponential stability of the system (15), is guaranteed by the uniform controllability condition of the system (1).

Lenmma 2 : If $(A(t), B(t))$ of the system (1) is uniformly controllable as shown by Eq.(5), the observability grammian $P_{1}(t)$ of the pair $\left(0, B^{T}(t) \Phi^{-T}\left(t, t_{0}\right)\right)$ satisfies

$$
\begin{gathered}
\frac{\beta_{1}}{m_{2}^{2}} I \leq P_{1}(t)= \\
\int_{t-\Delta}^{t} \Phi^{-1}\left(\tau, t_{0}\right) B(\tau) B^{T}(\tau) \Phi^{-T}\left(\tau, t_{0}\right) d \tau \leq \frac{\beta_{2}}{m_{1}^{2}} I
\end{gathered}
$$

where $\beta_{i}$ 's are as in Eq.(5) and $m_{i}$ 's as in Eq.(2).

Proof: Comparing the observability grammian $P_{1}(t)$ in Eq.(16) with the controllability grammian $P_{c}(t)$ of the system (1) in Eq.(5) shows that $P_{1}(t)=\Phi\left(t_{0}, t-\right.$ $\Delta) P_{c}(t) \Phi^{T}\left(t_{0}, t-\Delta\right)$. Hence, given any constant vector $x$, one has

$$
\beta_{1}\left\|\Phi^{T}\left(t_{0}, t-\Delta\right) x\right\|^{2} \leq x^{T} P_{1}(t) x \leq \beta_{2}\left\|\Phi^{T}\left(t_{0}, t-\Delta\right) x\right\|^{2},
$$

due to Eq.(5). Since $\Phi\left(t_{0}, t-\Delta\right)=\Phi^{-1}\left(t-\Delta, t_{0}\right)$, it follows from Eq.(3) that

$$
\frac{1}{m_{2}}\|x\| \leq\left\|\Phi^{T}\left(t_{0}, t-\Delta\right) x\right\| \leq \frac{1}{m_{1}}\|x\| .
$$

Therefore,

$$
\frac{\beta_{1}}{m_{2}^{2}}\|x\|^{2} \leq{ }_{2}{ }^{T} P_{1}(t) x \leq \frac{\beta_{2}}{m_{1}^{2}}\|x\|^{2} .
$$

End of proof.

The following theorem, which is the main result of this paper, shows that the proposed control (14) ensures exponential stability of the closed-loop system.

Theorem 2 : Consider the system (1) and the state feedback control (14). If the system (1) is uniformly controllable, and the open-loop state transition matrix satisfies the inequalities (2), the closed-loop system state $x(t)$ converges to zero exponentially.

Proof : Based on Theorem 1, one concludes immediately from Eqs.(15) and (16) that the transformed state $z(t)$ decays to zero exponentially in the sense that

$$
\|z(k \Delta)\|^{2} \leq \rho^{k}\|z(0)\|^{2}, \quad k=1,2, \ldots,
$$

where $0<\rho<1$, and

$$
\rho=1-2 \sigma \gamma, \quad \sigma=\frac{m_{1}^{4} \beta_{1}}{m_{2}^{2}\left(m_{1}^{2}+\gamma \beta_{2} \sqrt{n}\right)^{2}},
$$

in which Eq.(17) is obtained from Eq.(10) with $\alpha_{1}=$ $\beta_{1} / m_{2}^{2}$ and $\alpha_{2}=\beta_{2} / m_{1}^{2}$. Since $\|x(t)\| \leq m_{2}\|z(t)\|$ due to Eqs.(2) and (12), $x(t)$ also converges to zero exponentially. End of proof.

\section{Observer Design}

In this section, it is assumed that the only accessible signal in the system (1) is the system output $y(t)$. In order to estimate the system state $x(t)$, a conventional Luenberger type observer is adopted:

$$
\begin{aligned}
\dot{\hat{x}}(t)= & A(t) \hat{x}(t)+B(t) u(t)+ \\
& L(t)(y(t)-C(t) \hat{x}(t)), \quad \hat{x}(0)=\hat{x}_{0},
\end{aligned}
$$

where $\hat{x}(t) \in R^{n}$ is an estimate of the system state $x(t)$, and $L(t) \in R^{n \times p}$ is the observer feedback gain to be determined so that $x(t)$ approach $x(t)$ exponentially. Denote the state estimution error by $\tilde{x}=\hat{x}-x$, and subtract Eq.(18) from Ii.c.(1) to yield the state estimation error dynamics

$$
\dot{\grave{x}}=[A(t)-L(t) C(t)] \tilde{x}
$$

To design the observer feedback gain $L(t)$, the same coordinate transformation as in the control design is applied to the estimation error dynamics (19): 


$$
\tilde{x}(t)=\Phi\left(t, t_{0}\right) \tilde{z}(t),
$$

where $\bar{z}(t)$ defines the new coordinate, and $\Phi\left(t, t_{0}\right)$ is as before the state transition matrix of $A(t)$. The governing equation of $\tilde{z}(t)$ then becomes

$$
\dot{\tilde{z}}=-\Phi^{-1}\left(t, t_{0}\right) L(t) C(t) \Phi\left(t, t_{0}\right) \tilde{z} .
$$

Theorem 1 suggests that the observer feedback gain $L(t)$ be chosen as

$$
L(t)=\nu \Phi\left(t, t_{0}\right) \Phi^{T}\left(t, t_{0}\right) C^{T}(t), \quad \nu>0,
$$

where $\nu$ can be any positive constant. The resultant estimation error dynamics becomes

$$
\dot{z}(t)=-\nu \Phi^{T}\left(t, t_{0}\right) C^{T}(t) C(t) \Phi\left(t, t_{0}\right) \tilde{z}(t),
$$

which again has the same structure as the parameter estimation error dynamics of the gradient algorithm in Eq.(7) with now the regressor being $\Phi^{T}\left(t, t_{0}\right) C^{T}(t)$.

The following lemma shows that this regressor $\Phi^{T}\left(t, t_{0}\right) C^{T}(t)$ is persistently exciting if the system (1) is uniformly observable.

Lemma 3 : If $(A(t), C(t))$ of the system (1) is uniformly observable as shown in Eq.(6), the observability grammian $P_{2}(t)$ of the pair $\left(0, C(t) \Phi\left(t, t_{0}\right)\right)$ satisfies

$$
\begin{aligned}
& \gamma_{1} m_{1}^{2} I \leq P_{2}(t)= \\
& \int_{t-\Delta}^{t} \Phi^{T}\left(\tau, t_{0}\right) C^{T}(\tau) C(\tau) \Phi\left(\tau, t_{0}\right) d \tau \leq \gamma_{2} m_{2}^{2} I
\end{aligned}
$$

where $\gamma_{i}$ 's are as in Eq.(6) and $m_{i}$ 's as in Eq.(2).

The proof of Lemma 3 duplicates that of Lemma 2 by noticing that $P_{2}(t)=\Phi^{T}\left(t-\Delta, t_{0}\right) P_{o}(t) \Phi\left(t-\Delta, t_{0}\right)$. With the persistent excitation condition (22), one can now conclude the exponential stability of the state estimation error dynamics (19) by quoting Theorem 1.

Theorem 3 : Consider the state estimation error dynamics (19) and (20). If the system (1) is uniformly observable, and the open-loop state transition matrix satisfies the inequalities (2), the state estimation error $x(t)-x(t)$ converges to zero exponentially.

Proof: The proof follows exactly that of Theorem 2, and is omitted.

Finally, it is remarked that when the only accessible signal is the system output $y(t)$, the state feedback control in Section 4 should be replaced by an observerbased state feedback control

$$
u(t)=-K(t) x(t)
$$

where $K(t)$ is as designed in Eq.(14), and $\hat{x}(t)$ is obtained from the observer (18). Under the uniform controllability and observability assumptions and by quoting Theorems 2, 3, and the well-known Separation Property [2], it can be shown that the closed-loop system state under the control (23) converges to zero exponentially.

\section{Conclusions}

This paper proposies a new control design for a class of linear time-varying systems whose state exhibits sustained but bounded oscillations. The new approach utilizes the gradient algorithm, which is originally used in the parameter identification problem, to find stabilizing control and observer feedback gains. It is worth mentioning that conventionally for a system with time-varying parameters, the observer feedback gain depends on past and present information of the time-varying parameters, and the control feedback gain, which is obtained as a dual result of the observer design, depends on future information of the time-varying parameters. However for the proposed design in this paper both the observer feedback gain and the control feedback gain depend only on past and present information of the time-varying parameters.

\section{References}

[1] S. Bittanti, P. Colaneri, and G. Guardabassi, "Analysis of the periodic Lyapunov and Riccati equations via canonical decomposition," SIAM. J. Control and Optimization, vol. 24, pp.1138-1149, 1986.

[2] C. T. Chen, Linear System Theory and Design, Holt, Rinehart and Winston, New York, 1984.

[3] R. A. Calico, and W. E. Wiesel, "Control of timeperiodic systems," J. of Guidance, vol. 7, pp.671$676,1984$.

[4] F. Callier and C. A. Desoer, Linear System Theory, Springer-Verlag, Hong Kong, 1992.

[5] O. Follinger, "Design of time-varying system by pole assignment," Ri gelungstechink, vol. 26, pp. 8595, 1978.

[6] H. Kano, and T. Nishimura, "Periodic solutions of matrix Riccati equations with dectectability and stabilzability," Int. J. Control, vol. 29, pp.471-487, 1979. 
[7] H. Kwakernaak and R. Sivan, Linear Optimal Control Systems, Wiley, New York, 1972.

[8] K. S. Narendra and A. M. Annaswamy, Stable Adaptive Systems, Prentice-Hall, Englewood Cliffs, New Jersey, 1989.

[9] W. J. Rugh, Linear System Theory, Prentice-Hall, Englewood Cliffs, New Jersey, 1993.

[10] S. Sastry, and M. Bodson, Adaptive Control, Stability, Convergence, and Robustness, Prentice-Hall, New York, 1989.

[11] S. C. Sinha, P. Joseph, "Control of general dynamic systems with periodic varying parameters via Liapunov-Floquet transformation," ASME J. of Dynamic Systems, Measurement, and Control, vol. 116, pp.650-658, 1994.

[12] M. Valasek, and N. Olgac, "Generalization of Ackermann's formula for linear MIMO time-invariant and time-varying systems," Proceedings of 1993 Conference on Decision and Control, pp. 827-831, 1993.

[13] W. A. Wolovich, "On the stabilization of controllable systems," IEEE Transaction on Automatic Control, pp. 569-572, 1968. 